\title{
Evaluation of Comfort in a Swinging Chair by Utilizing Brainwaves and Heartbeat Fluctuation
}

\author{
Keiji Matsumoto ${ }^{\mathrm{a}^{*}}$ \\ aNational Institute of Technology, Kitakyushu College 5-20-1 Shii, Kokuraminami, \\ Kitakyushu, Fukuoka, 802-0985, Japan \\ "Corresponding Author:kmatsu@kct.ac.jp
}

\begin{abstract}
In this paper, we propose a swinging chair that provides comfortable oscillation. It has a distinct characteristic of automatically producing a repetitive back-and-forth reciprocating motion based on a signal generated using fluctuation obtained from a person's heartbeat. Brainwaves and heartbeats are employed to evaluate comfort, as opposed to the widely used, questionnaire-based factor analysis. The Index of Brain Resting State (IBRS) calculated using measured brainwaves is used as an index of the brainwave. The area of an ellipse, the value calculated by multiple regression equation, and the value of $T / L$ (the ratio of between the major axis $L$ of the data on Lorentz plot and the minor axis $\mathrm{T}$ of the data on Lorentz plot) are used as indices of heartbeat fluctuation. This time, the degree of comfort is evaluated using this bio-information, and the results are reported.
\end{abstract}

Keywords: heartbeat, Lorentz plot, brainwave.

\section{Introduction}

As a result of the wonderful development of information and communications technology (ICT) and internet of things (IoT) in recent years, people in modern societies are living a comfortable and abundant life. However, the spread of these technologies has led to global competition among companies and cost reduction in employee's personnel. Consequently, people's stress levels are augmenting at an unprecedented rate. In recent years, research and development with respect to amenity and sensibility has been observed in various fields to address these problems [1-3]. The purpose of research on amenity is mainly to quantify the notion of sensory aspect.
It requires insight beyond the realms of learning. The fields of research in regard to amenity are largely classified into four categories [4].
1. Living comfort
2. Ride quality
3. Sitting comfort of a chair
4. Clothing comfort

Living comfort is believed to be of foremost interest amongst these, for releasing daily stresses. In a typical living environment, the comfort is created through light, sound, and temperature, while the vibrational element is overlooked as a disagreeable element. However, the category of vibration includes both unpleasant and pleasant elements. An infant's cradle has wonderful features that provide comfort. See-saws, swings, and hammocks are practical examples of products that use swinging to release stress. Moreover, comfortable vibration in a car makes people lethargic. The active rocking chair has been developed that has employed this oscillation characteristic successfully [5-6]. In this study, the comfort of a rocking chair that is intrinsically controlled using a person's heartbeat fluctuation is evaluated using a questionnaire. The questionnaire evaluates parameters representing the psychological state of human beings on a numerical scale with five levels. However, the evaluated numerical value does not necessarily represent the psychological state as the humans' sense is relatively ambiguous.

In this study, we propose a swinging chair that provides comfortable oscillation. It has a distinct characteristic of automatically producing a repetitive back-and-forth reciprocating motion based on a signal generated using fluctuation obtained from a person's heartbeat. We produced a prototype of the swinging chair, and then presented and reported it as new comfortable equipment [7]. This time, the degree of comfort is evaluated in the modified swinging chair using this bio-information, 
and the results are reported.

\section{Method}

\subsection{Structure of the swinging chair}

A typical chair has either a fixed seat or a rotating seat. We believe that a comfortable chair can be realized by modifying a common chair. Fig.1 shows the structure of the swinging chair. The swinging chair pleasantly oscillates, and its range of motion is limited to $360^{\circ}$. Fig. 2 shows the fabricated swinging chair and the structure of the rotating part. The rotating mechanism consists of a motor bracket, a pulley, and a timing belt. The driving motor is fixed on the bracket installed on the chair body. The pulley of diameter $120 \mathrm{~mm}$ is attached to the motor shaft. The axis rotating in synchronization with the chair seat has the same diameter as that of the pulley. The power from the motor is transmitted to the chair seat through the toothed belt that connects both the pulleys. A small DC motor of approximately $60 \mathrm{~W}$ is used to drive this mechanism. This motor is operated by the control box of the swinging chair. Fig.3 shows the fabricated control box. The control box primarily consists of a heartbeat sensor, an Arduino, and a motor driver. The heartbeat waveform is transformed into the corresponding voltage using the heartbeat sensor attached to the human. This voltage is transmitted to the Arduino. The Arduino computes the operation time of the swinging chair using the heartbeat fluctuations. The signal is transmitted from the Arduino to the power circuit board and amplified. Subsequently, the rotating motion is realized.

\subsection{Method to create an oscillation with fluctuation}

In this study, the average heartbeat period of a human is believed to be the criterion, and the difference between each heartbeat period and the average value is the heartbeat fluctuation. Kawashima et al. reported the comfortable vibration frequencies through an experiment with a rocking chair that operated at various frequencies using a motor [5, 6]. Last time, we produced a prototype of the swinging chair, and then presented and reported it as new comfortable equipment [7]. The proposed driving algorithm in the previous report was not used to vary the driving speed, but to change the operating time depending on the heartbeat fluctuation. In this study, we examine the way to realize a comfortable feel by giving people an obvious perception using fluctuation of rotational rate. Fig. 3 shows a command value for the rotational motion that changes with each heartbeat fluctuation. When the heartbeat fluctuation decreases due to the predominance of sympathetic nerve activity, the system slowly drives the swinging chair. In contrast, when the heartbeat fluctuation increases due to the predominance of parasympathetic nerve activity, the system drives it faster. It was confirmed through an experiment that too much of high rotational motion is uncomfortable; thus, the maximum revolution speed is limited to $1.08 \mathrm{rad}$ per sec. To avoid a sudden change in motion and preserve comfort, an S-shaped curve shown in Fig. 3 is adopted to the acceleration waveform.

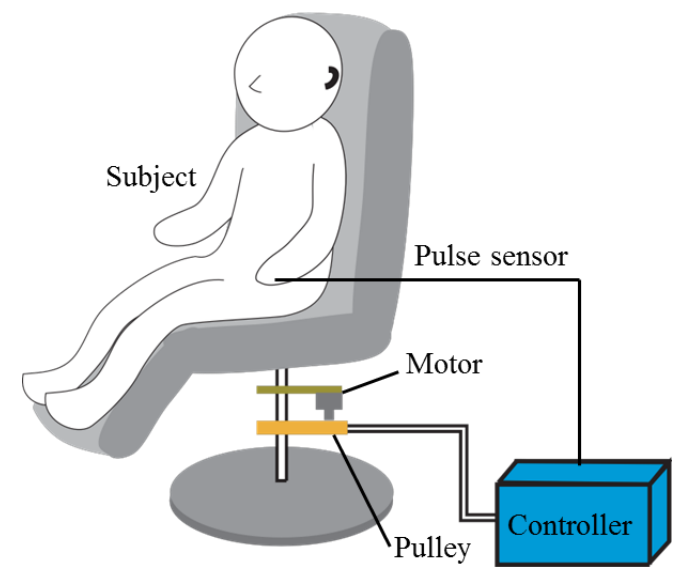

Fig.1 Structure of the swinging chair

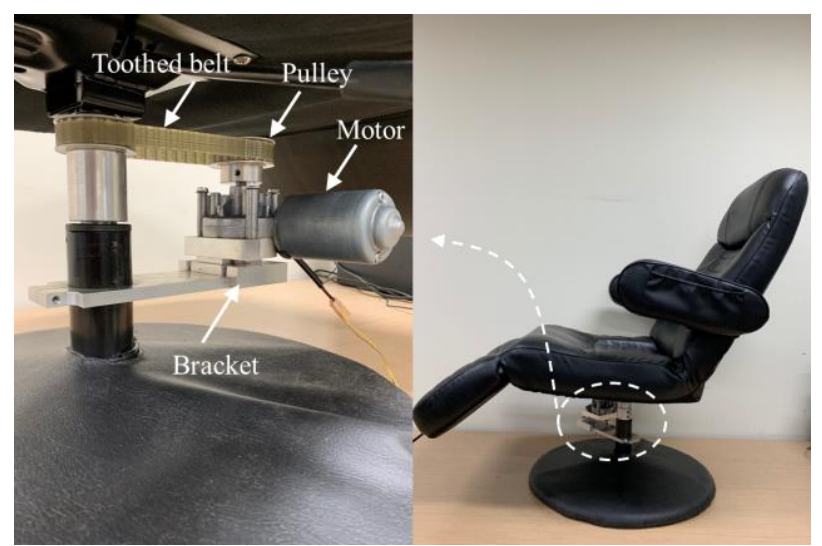

(1)

(2)

Fig.2 (1) Appearance and (2) rotating mechanism of the swinging chair 


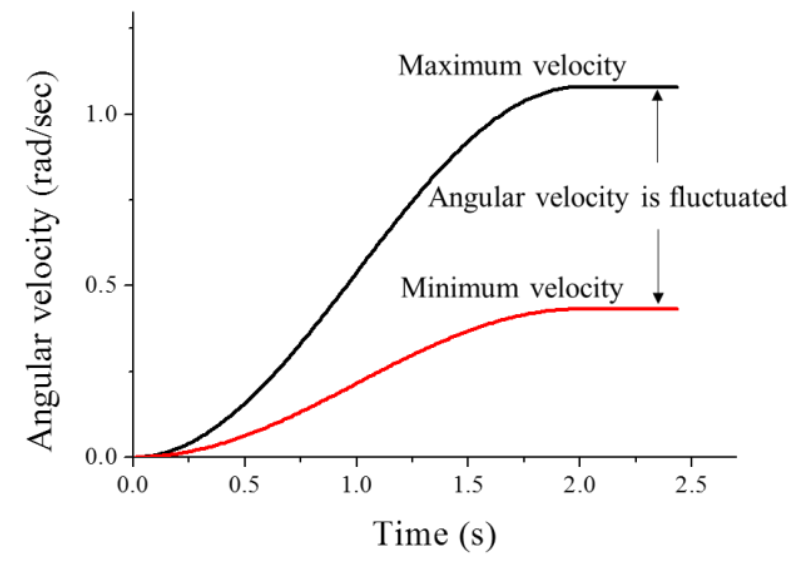

Fig.3 Control signal modulated by the fluctuation

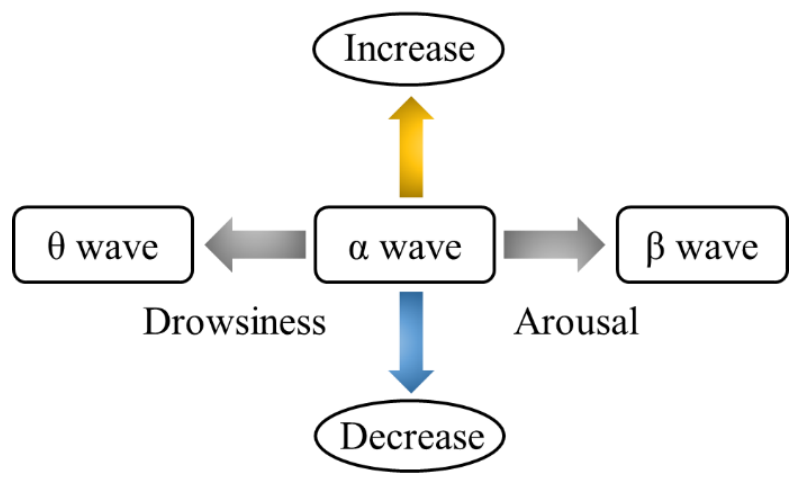

Fig.4 Relationship between the human state and brainwave frequencies

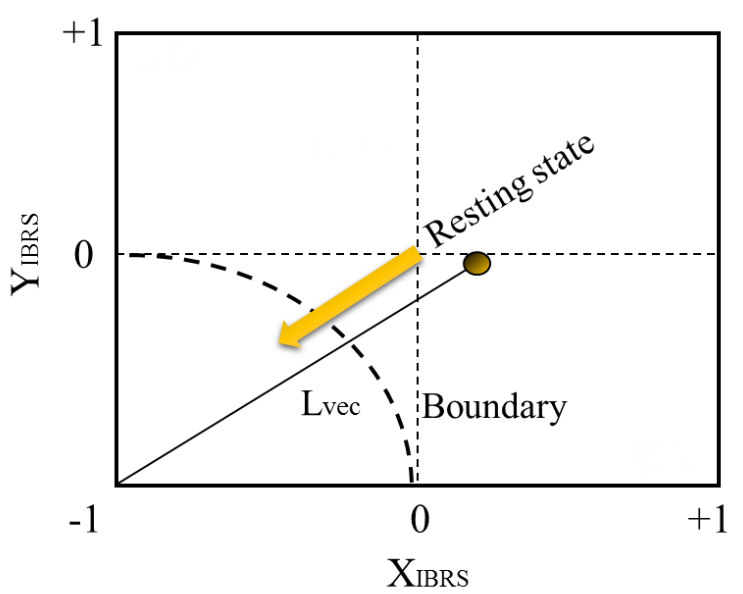

Fig.5 2D display of the IBRS

\subsection{Evaluation method}

In order to evaluate comfort, a questionnaire followed by factor analysis using the semantic differential method is widely used [8]. The subject's impression is expressed by numerical of about 5 levels for several items and the obtained value is used as a psychological feature. However, the evaluated numerical value does not necessarily represent the psychological state. Thus, in this study, the psychological feature is evaluated using the brainwaves and heartbeat fluctuation that are bio-information related to the human psychological state [9]. The IBRS calculated by a measured brainwave is used as an index of the brainwave. An area of an ellipse, a value calculated by multiple regression equation, and value of $\mathrm{T} / \mathrm{L}$ are used as an index of the heartbeat fluctuation.

\subsection{IBRS}

IBRS is an evaluation method for the resting state of human based on an electroencephalographic index [10]. Fig. 5 shows the conceptual diagram of the human state and brainwave frequencies. Generally, the alpha wave is used as an index of resting, and its integral amplitude value is ordinarily used as the discrimination criterion. However, other brainwave information is also needed to correctly estimate the human state, because alpha waves are considerably affected by visual information. Generally, the beta-wave element increases, along with its frequency, if the brain enters an excited state. In contrast, when the brain becomes drowsy and enters a suppressed state, the thetawave element increases, and its frequency decreases. The condition between arousal and drowsiness is considered to be the brain-resting state. Therefore, the amplitude of the alpha wave compared to those of the theta and beta waves is defined as the IBRS. Formulas for the IBRS are defined as follows.

$$
\begin{gathered}
X_{I B R S}=\frac{\theta_{a m p}-\alpha_{a m p}}{\theta_{a m p}+\alpha_{a m p}} \\
Y_{I B R S}=\frac{\beta_{a m p}-\alpha_{a m p}}{\beta_{a m p}+\alpha_{a m p}} \\
L_{\text {vec }}=\sqrt{\left(1+\frac{1}{n} \sum_{i=1}^{n} X_{i}^{I B R S}\right)^{2}+\left(1+\frac{1}{n} \sum_{i=1}^{n} Y_{i}^{I B R S}\right)^{2}}
\end{gathered}
$$

The IBRS is visually indicated in two dimensions using the ratio between the theta and alpha waves or between the beta and alpha waves (Fig.6). The horizontal axis is defined as 
the IBRS' $X$ component, which is represented by both the theta and alpha waves, and the vertical axis is defined as the IBRS' Y component, which is represented by the beta and alpha waves. Both components of the IBRS are represented in a range of -1 to +1 . The value gradually becomes closer to -1 as the alpha wave increases. When the point $(-1,-1)$ is assumed to be the origin, the resting rate increases when the point indicated as the IBRS coordinate moves closer to the origin. The vector length from the origin is defined as the IBRS, as in equation (3). We estimated the human state using this index.

\subsection{Indices extracted from the heartbeat fluctuation}

Fig.6 shows an example of a heartbeat waveform. The peak is called $R$ wave, the space between the $R$ waves is called the R-R interval (RRI). The fluctuation of RRI, which is influenced by the sympathetic and parasympathetic nerve activities, is called heartbeat fluctuation. Recently, the Lorentz plot (LP) has become a widely used method to evaluate the heartbeat fluctuation. In the LP method, the value of RRI of nth and the value of RRI of $(n+1)$ th are plotted on the horizontal and vertical axis respectively. Under active state, the data on the LP tend to be compacted, as heartbeat fluctuation decreases due to sympathetic nerve activity. In contrast, the data under the resting state tend to be scattered on the LP, as heartbeat fluctuation increases due to parasympathetic nerve activity. Several ways to evaluate the human state using this feature have been proposed. Fig.7 shows the method to evaluate the RRI on the LP. First, project all the plotted data on the LP to the $y=x$ axis and $y=-x$ axis. The variability on the $y=x$ axis and the variability on the $\mathrm{y}=-\mathrm{x}$ axis are named $\delta \_\mathrm{x}$ and $\delta \_(-x)$ respectively. The distance between the mean value of all the data on LP and origin $(0,0)$ is named $\mathrm{m}$, and the area of the ellipse is expressed as

$$
S=\pi \times \delta_{x} \times \delta_{-x}
$$

The method to evaluate the state of parasympathetic nerve activity by means of the area of the ellipse is widely used [11]. It was proposed that the value calculated by multiple regression analysis (using both the $\mathrm{m}$ and the area $\mathrm{S}$ is as a criterion to evaluate the parasympathetic nerve activity) is expressed as

$$
y=0.592 \times \mathrm{m}+0.238 \times S
$$

Moreover, the $\mathrm{T} / \mathrm{L}$ ratio of between the major axis $\mathrm{L}$ of the data on LP and the minor axis T of the data on LP is also widely used as a criterion of the parasympathetic nerve activity [12]. In this study, these 3 methods are employed to evaluate the heartbeat fluctuation.

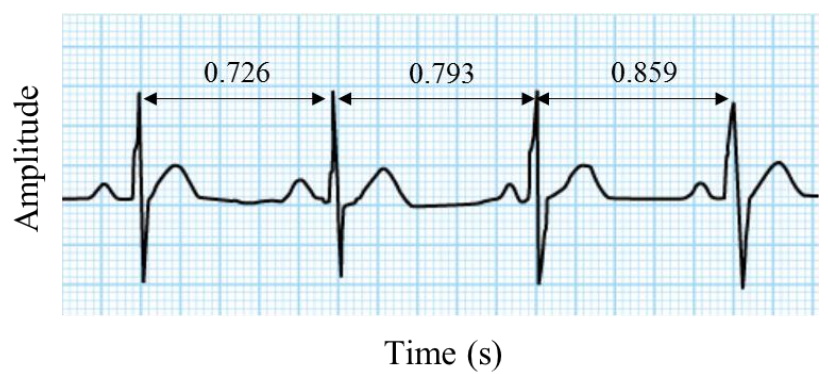

Fig.6 Heartbeat waveform in the time domain

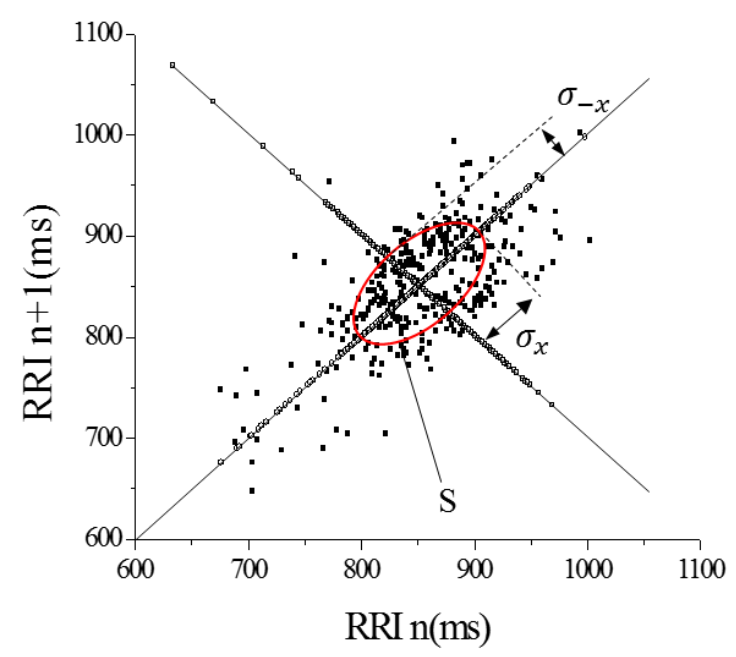

Fig.7 Evaluation method on Lorentz plot

\subsection{Experimental procedure}

The subjects wearing the EEG and heartbeat sensor sit on the swinging chair and relax. Once the measurement begins, the swinging chair begins oscillating. The measurement is performed for $5 \mathrm{~min}$. The brainwave is detected by the EEG sensor and transmitted to a desktop computer for analysis during the measurement. The heartbeat measured by the heartbeat sensor is received at the Arduino. The subject's state is evaluated by means of both the IBRS calculated using the measured brainwaves and the parameters calculated by measured heartbeat once the experiment is completed. 


\section{Result and discussion}

Three types of experiments about the swinging chair of no operation, no fluctuation and with fluctuation were conducted and the brainwaves and the heartbeat fluctuation were acquired. First, the heartbeat fluctuation was examined. Fig. 8 shows the standardized ellipse area $\mathrm{S}$ representing the scattering data on the LP. In case of no operation, the smallest value (in all of them) was -0.76. In case of no fluctuation and with fluctuation, the values were -0.37 and 1.13 respectively. It is assumed that the latter state was dominated by parasympathetic nerve activity due to it being the largest area of the ellipse. Next, the values calculated by equation (5) were examined. Fig.9 shows the standardized values obtained from equation (5). The values of no operation, no fluctuation, and with fluctuation were $-0.74,-0.39$ and 1.14 respectively. As the parasympathetic nerve activity was thought to be the dominant one, 'with fluctuation' produced the biggest value of all. The final process of heartbeat fluctuation analysis is T/L. Fig.10 shows the standardized respective T/L. The values of no operation, no fluctuation, and with fluctuation were -0.79 , -0.34 , and 1.13 respectively. Even in T/L, 'with fluctuation' is assumed to be the most dominant state in terms of parasympathetic nerve activity. Three analyses of the ellipse area, multiple regression equation, and $\mathrm{T} / \mathrm{L}$ showed a similar tendency. 'No fluctuation' showed a bigger value than 'no operation' did. 'With fluctuation' showed a bigger value than 'no fluctuation'. Regardless of the indices used for comparison, 'with fluctuation' was the most dominant state in terms of parasympathetic nerve activity. Next, the measured brainwaves were examined. Fig.11 shows standardized respective IBRS. 'No operation' produced -1.10 , the smallest value of all, while 'no fluctuation' and 'with fluctuation' produced 0.24 and 0.86 respectively. The IBRS of 'with fluctuation' produced the biggest value meaning the largest appearing alpha wave comparing with the beta wave and the theta wave. Based on the aforementioned results, all types of indices (both the brainwaves and the heartbeat fluctuations) showed similar tendencies in all three cases. To examine the repeatability of the performance, the case of 'with fluctuation' was conducted several times. The brainwave and heartbeat fluctuation were acquired five times under the state of 'with fluctuation', and the mean values of these measurements were compared with the other case's values. Fig.12 shows the comparison of values from the ellipse area, the multiple regression, the $\mathrm{T} / \mathrm{L}$ and the IBRS.

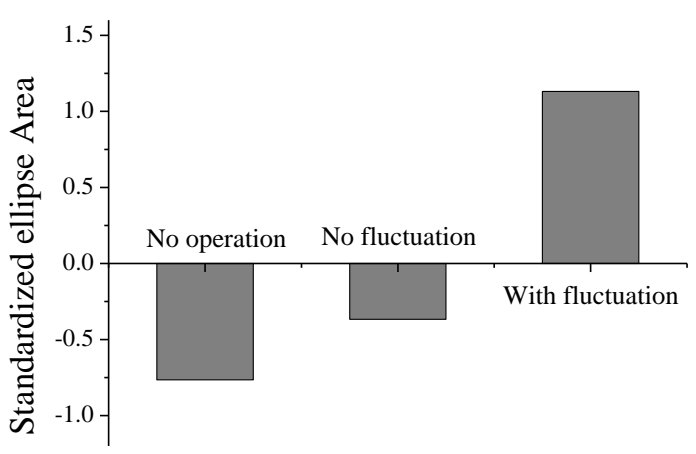

Fig.8 Standardized value of ellipse area in three cases

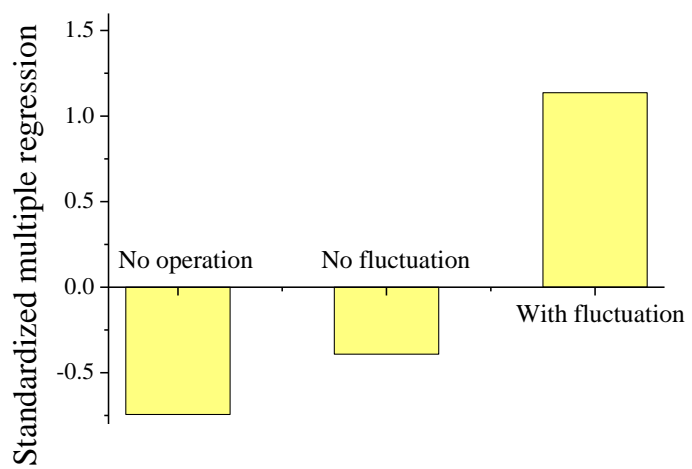

Fig.9 Standardized value of multiple regression in three cases

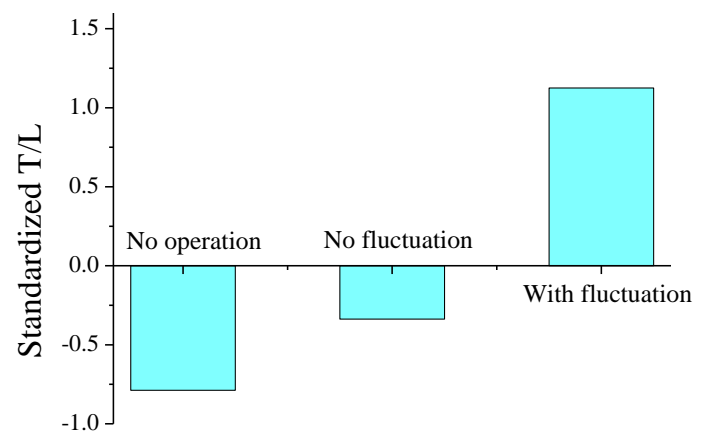

Fig.10 Standardized value of T/L in three cases

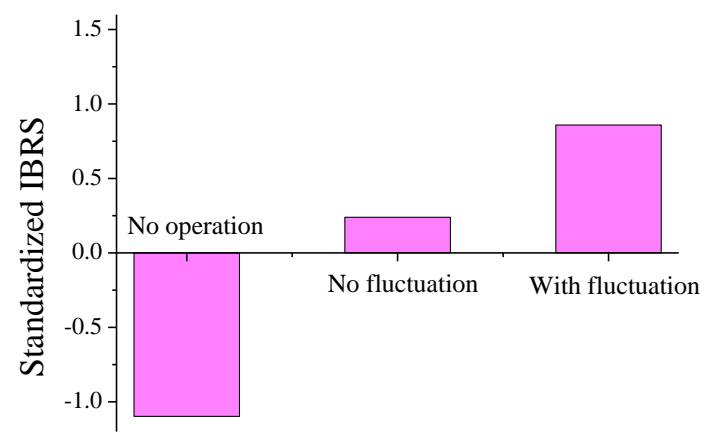

Fig.11 Standardized value of IBRS in three cases 


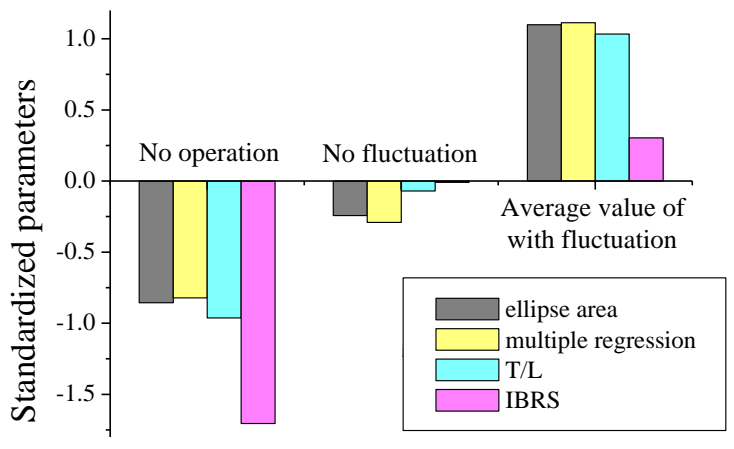

Fig.12 Standardized value of each parameter in three cases

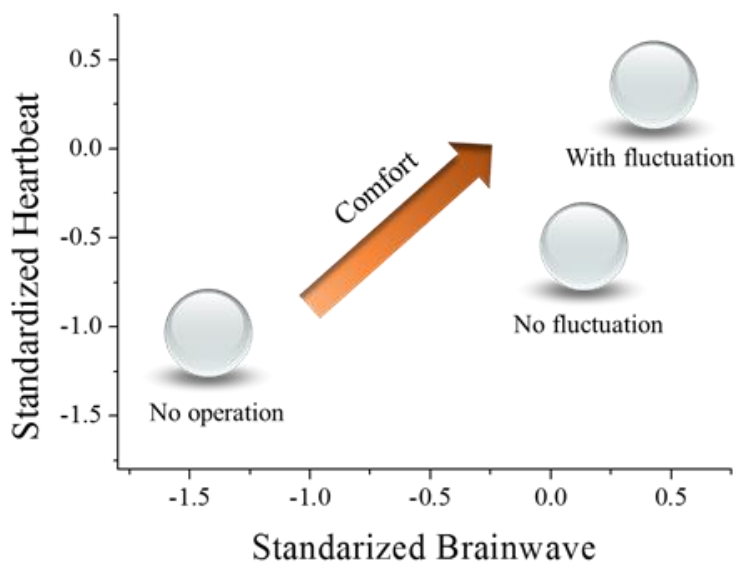

Fig.13 Tendency of parasympathetic nervous activity

Throughout the repeated occurrences, the tendency remained the same. The case of 'no operation' produced the lowest value of all. In contrast, the case of 'with fluctuation' produced the highest. Under this experimental condition, the repeated occurrences of parasympathetic nerve activity were found to be in 'with fluctuation'. Fig.13 shows a tendency of parasympathetic nerve activity based on brainwaves and heartbeat fluctuations. The vertical axis shows the mean value of the standardized ellipse area, multiple regression, and $\mathrm{T} / \mathrm{L}$. The horizontal axis shows standardized IBRS. On this graph, as it positions in the upper right direction, it becomes more dominant of parasympathetic nerve activity. As the value of both axes increases, the relaxation effect tends to be higher. Therefore, it is thought that the swinging chair that produces a back-and-forth reciprocating motion based on the signal generated using heartbeat fluctuation, provides a relaxation effect and has a possibility to help people release stress.

\section{Conclusions}

This time, the degree of comfort was evaluated using the indices of bio-information, and the results were reported. Three analyses of the ellipse area, multiple regression equation, and T/L showed a similar tendency. Regardless of the indices used for comparison, 'with fluctuation' was the most dominant state in terms of parasympathetic nerve activity. The IBRS of 'with fluctuation' produced the biggest value meaning the largest appearing alpha wave comparing with the beta wave and the theta wave. Throughout the repeated occurrences, the tendency remained the same. Based on the aforementioned results, all types of indices (both the brainwaves and the heartbeat fluctuations) showed similar tendencies in all cases. This experiment was carried out with one participant because evaluating the comfort of the swinging chair using various indexes was main focus. From now on, an evaluation of the data collected with lots of participants is very important task. However, it is thought that the swinging chair that produces a back-and-forth reciprocating motion based on the signal generated using heartbeat fluctuation, provides a relaxation effect and has a possibility to help people release stress.

\section{References}

(1) T.Kotani, H.Higashi, "Approach for Realization of Comfortable Lighting Environment Using LED Luminaires", Toshiba review, Vol.65, No.7, pp.20-23, 2010

(2) B.Ko, D.Lee, H.Chiang, T.Koga, and K.Hirate, "Basic Study on Rest Effect of Light Color in the LED Lighting", J. Environ. Eng. AIJ, Vol.76, No.662, pp.363-368, 2011

(3) F.Wang, K.Sagawa \& H.Inooka, "A study of the relationship between the longitudinal acceleration/deceleration of automobiles and ride comfort", human engineering, Vol.36, No.4, pp.191-200, 2000

(4) K.Suzuki, "Measure comfort: evaluation of its psychological, behavioral and physiological effects, Japan Publication Service, 2002, in Japanese

(5) T.Kawashima, "Study for Comfortable Swinging: Comfortable Swinging for Active Rocking Chair", The Japan Society of Mechanical Engineers, Vol.69, No.677, pp.219-226, 2003 
(6) T.Kawashima, "Development of Active Rocking Chair Changing the Swing with Heartbeat Fluctuation", The Japan Society of Mechanical Engineers, Vol.71, No.709, pp.2731-2737, 2005

(7) J.Eto, R.Yagami, T.Shimosako, T.Sokena, K.Matsumoto, "Comfortable Oscillation in Swinging Chair Using Heartbeat Fluctuation", Proceedings of the 4th IIAE International Conference on Intelligent Systems and Image Processing 2017, pp.140-145, Sep, 2017

(8) M.Nagamachi, "Kansei engineering: technology of human senses in design (In Japanese)", Kaibundo Publishers, 1989

(9) T.Ichikawa, "An Invitation to Journey for Understanding of EEG", Seiwa Shoten Publishers, 2006, in Japanese
(10) Kaoru Yoshii and Keiji Matsumoto, "A Dry-Eye Warning System Based on the Estimation of the Human State Using a Single-Channel EEG Sensor", Proceedings of the 4th IIAE International Conference on Intelligent Systems and Image Processing 2016, pp.142-148, Sep, 2016

(11)F.Toyofuku, K.Yamaguchi, and H.Hagiwara, "Simplified method for estimating parasympathetic nervous activity by Lorenz plot of ECG RR intervals", ergonomics society, Vol.43, No.4, pp.185-192, 2007

(12) N.Dodo, "Effects of Sound Tempos on Autonomic Nervous System Functions", J Psychol Sci, No.8, pp.7-13, 2012 